Communications in Physics, Vol. 30, No. 3 (2020), pp. 245-256

DOI:10.15625/0868-3166/30/3/14834

\title{
MICROFLUIDIC CHIP FOR TRAPPING MAGNETIC NANOPARTICILES AND HEATING IN TERMS OF BIOLOGICAL ANALYSIS
}

\author{
LE NGOC TU ${ }^{1,2}$, NGUYEN CONG THINH $^{3}$, TRAN DAI LAM ${ }^{2,4}$, NGUYEN VAN-ANH $^{5}$ \\ AND CAO HONG HA ${ }^{5 \dagger}$
}

${ }^{1}$ National Psychiatric Hospital No. 1, Thuong Tin, Hanoi, Vietnam

${ }^{2}$ Graduate University of Science and Technology, Vietnam Academy of Science and Technology, 18 Hoang Quoc Viet, Hanoi, Vietnam

${ }^{3}$ Department of Science, Technology and Environment, Ministry of Construction, Vietnam

${ }^{4}$ Institute for Tropical Technology, Vietnam Academy of Science and Technology, 18 Hoang Quoc Viet, Hanoi, Vietnam

${ }^{5}$ School of Chemical Engineering, Hanoi University of Science and Technology, 1 Dai Co Viet, Hanoi, Vietnam

${ }^{\dagger} E$-mail: ha.caohong@hust.edu.vn

Received 18 February

Accepted for publication 4 May 2020

Published 24 July 2020

\begin{abstract}
In this study, we reported the results of the design and the fabrication a planar coil in copper (square, $a=10 \mathrm{~mm}, 15 \mu \mathrm{m}$ high, 45 turns), these planar coils were integrated in a microfluidic chip for trapping magnetic nanoparticles and local heating applications. A small thermocouple (type K, $1 \mathrm{~mm}$ tip size) was put directly on top of the micro-channel in poly(dimethyl-siloxane) in order to measure the temperature inside the channel during applying current. The parameters design of planar coils was based on optimizing the results of the magnetic field calculation by ANSYS ${ }^{\circledR}$ software. The $2 D$ simulation model was used for calculated the magnetic strength at different distances from the coil surface. These results shown that the magnetic field is stronger at the closer distance to the coil surface (magnetic field strength $H_{\max }$ is around $825 \mathrm{~A} / \mathrm{m}$ ). These results were also the basic for defining the suitable distance from the coil surface to the bottom of the micro-chanel of the microfluidic chip. The magnetic induction $(B)$ and heating relationship was balanced in order to manipulating the trapping magnetic nanoparticles and heating process. This design of the microfluidic chip can be used to develop a complex microfluidic chip using magnetic nanoparticles.
\end{abstract}

Keywords: microfluidic; planar-coil; magnetic nanoparticles; electromagnet; magnetic field calculation; Polydimethyl-siloxane.

Classification numbers: 75.75.Jn; 41.20.Jb.

C2020 Vietnam Academy of Science and Technology 


\section{INTRODUCTION}

Microfluidic chips have attracted interests of scientists due to the promise to revolutionize modern laboratories [1-3]. These devices possess potential to fundamentally change the way in which biological/chemical analysis is performed. Scientists expect that these devices will be a trend to miniaturize and automate the laboratory, a Lab - on - a Chip, and carry out multiple processes of biology and chemistry onto tiny chips... [4]. The different fluids of chemicals can be pumped into the micro-channel of the only one microfluidic system, and many processes such as mixing, separating, reacting and chemical sensing are manipulated inside micro-channel. Concerning the application of the microfluidic chip in biological analysis, such as the standard immunoassay (ELISA), the microfluidic immunoassay is mainly performed basing on bead-based immunoassay. It means that, the antibodies and antigens can be immobilized on the surface of the micro-channels or beads $[5,6]$.

To trap and move magnetic nanoparticles, permanent magnets can be used [7,8]. However, these magnets have a very limited controlling magnetic field, and there was no technology to enable fast and reversibly to manipulate magnetic particles. Besides, permanent magnets cannot be easily integrated in a lab-on-chip at several $\mu \mathrm{m}$ level of a channel width. Although the permanent magnet can generate strong magnetic intensity, i.e. few hundreds mT $[9,10]$, they are more difficult to operate flexibly for trapping and releasing magnetic particles in the channels. Recently, microelectromagnets have been used as a magnetic micro-actuator to manipulate magnetic nanoparticles in micro-channels, and a microfluidic system can be a digital chip for many applications [11,12].

Using the planar coil/micro-electromagnet that was integrated into microfluidic channel, biological agents can interact to the bio-functionalized surface of magnetic nanoparticles at the trapping area of the micro-channel. This trapping/releasing magnetic nanoparticles process can be independently controlled $[13,14]$. In parallel of the magnetic generation function of the planar coil, the heating function of it is activated by the Joule effect [15]. In a biological analysis process, the management of many stages of the physical state, biological and chemical reaction at suitable temperature is a critical condition, such as the PCR process $[15,16]$.

In this study, we propose designing and fabricating a microfluidic chip integrated with a copper planar coil, and the microfluidic chip was characterized via properties in trapping/releasing magnetic nanoparticles inside channel. In addition, the planar coil played a role of heating component.

\section{MATERIALS AND METHODS}

\section{II.1. Magnetic field calculation}

A model of coil was built to carry out the magnetic field simulation using Finite Element (FE) method thanks to ANSYS ${ }^{\circledR}$ v12.1 software. The criterion for designing planar coils was an optimal magnetic field of coil and the heating function in the microfluidic chip.

A conducting wire carrying a current, $I$, produced a magnetic field strength $\boldsymbol{H}$. The strength and direction of $\boldsymbol{H}$ depended on $r$, the distance from current to a point $\mathrm{P}$, as shown in 1 . The magnetic field at any point $P$ due to the current could be calculated by adding up the magnetic field contributions, $d \boldsymbol{H}$, from small segments of the wire $d \ell$, (see Fig. 1).

The Biot-Savart law (for infinite/straight wire) gives an expression for the magnetic field contribution, $d \boldsymbol{H}$, from the current source, $I . d \boldsymbol{\ell},[17]$ : 


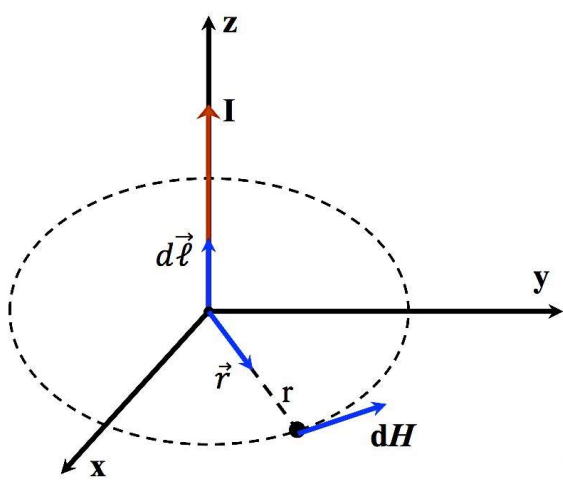

(a)

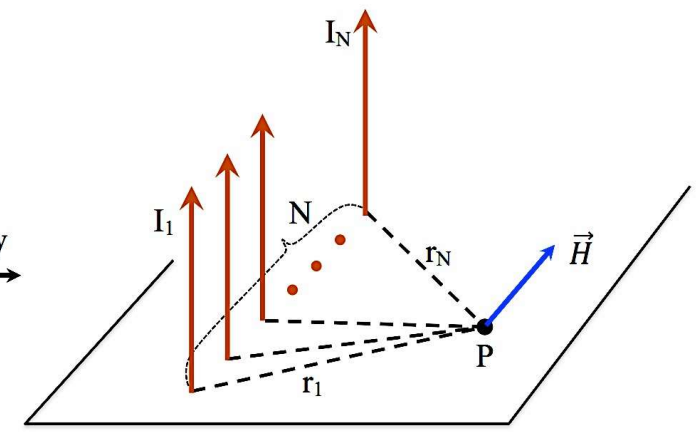

(b)

Fig. 1. (a) magnetic field strength $\boldsymbol{H}$ at point $\mathrm{P}$ due to a current-carrying element $\mathrm{Id} \ell$; (b) total magnetic field strength $\boldsymbol{H}$ at point $P$ due to $N$ parallel current-carrying element $I$.

$$
d H=\frac{1}{4 \pi} \frac{I d \ell \times \mathbf{r}}{r^{2}}
$$

in which $I$ is the current in the wire; $r$ is the distance from the wire to point $P ; r$ is the corresponding unit vector.

In case of rectangular or square coils, the distance to the considered point is not constant (Fig. 2).

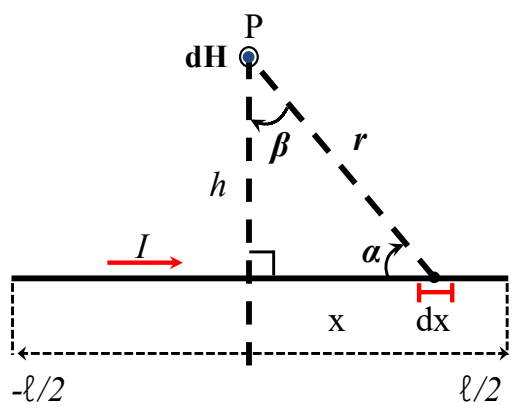

(a)

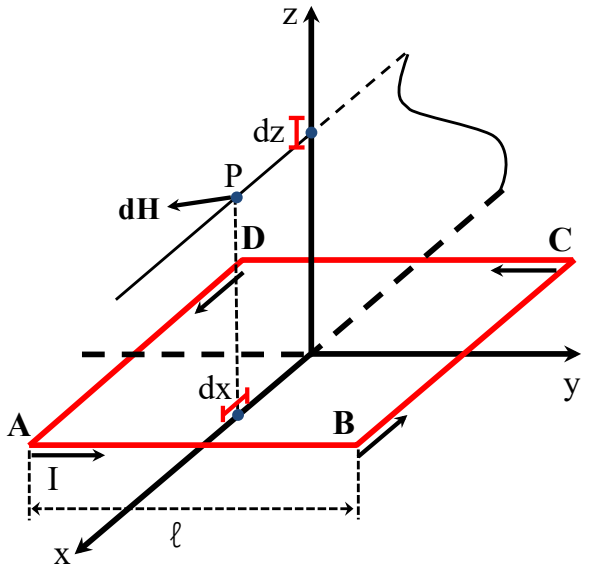

(b)

Fig. 2. The magnetic field strength $\boldsymbol{H}$ at point $\boldsymbol{P}$ inducing by the non-finite straight wire (a), and one square coil (b). I: the current; h: the distance from conductor wire to the point $\mathrm{P}$; $\mathrm{r}$ : the distance from the wire to point $\mathrm{P}$; $\ell$ : the edge length of the square turn. 
The elementary magnetic field strength $d \boldsymbol{H}_{i}$ (for one straight wire) produced by a small section $d x$ of the wire is:

$$
d H_{i}=\frac{1}{4 \pi} \frac{I \cos (\theta) d x}{r^{2}}=\frac{1}{4 \pi} \frac{I \cos (\theta) d \theta}{r^{2}}
$$

For the square coil (including 4 single straight wires):

$$
H=4 \times H_{i}
$$

The magnetic field generated by a portion of single wire of square coil can be calculated using Eq. (1). In the case of multiple turns of a coil, the magnetic field is defined as the vector sum of each wire segment component generated magnetic field strength $\boldsymbol{H}_{i}$. given by:

The relationship between the magnetic field strength $\boldsymbol{H}$ and the magnetic induction $\boldsymbol{B}$ is

$$
\boldsymbol{B}=\mu_{0} \cdot \boldsymbol{H} \cdot\left(1+\chi_{m}\right)=\mu_{0} \cdot \mu_{r} \cdot \boldsymbol{H}
$$

where $\chi_{m}$ is the magnetic susceptibility of material, $\mu_{0}$ is the permeability of free space: $\mu_{0}=$ $4 \pi \times 10^{-7}$ T.m/A. In the case of vacuum, water or air, $\mu_{r}=1+\chi_{m}=1$.

The merit factors such as the magnetic field generated for a given power loss are defined. The effective coil for magnetic beads trapping can be found with narrow wires and large number of turns. Furthermore, the known efficient trapping area close to the coil surface (around $10 \mu \mathrm{m}$ ) will be observed.

\section{II.2. Fabrication of planar coil}

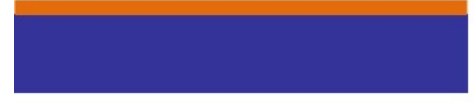

1.Depositing Metallic seed layer on Si wafer

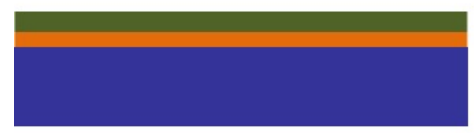

2.Spin-coating photoresist

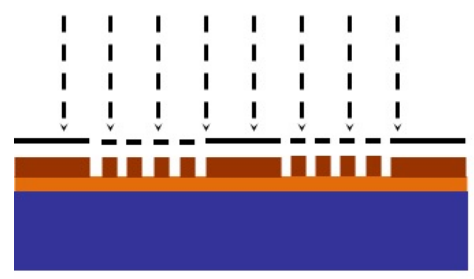

3.UV-exposure with photomask

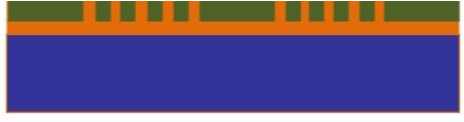

4.Cu electro-deposition

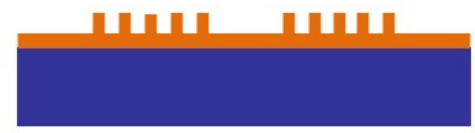

5.Photoresist removal

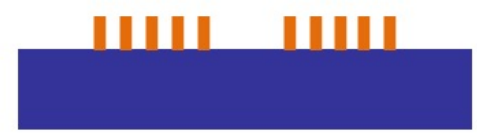

6.Etching seed layer

Fig. 3. The fabrication processes of the planar coil. 
The planar coil was fabricated micro fabrication technique in clean room as following steps, as shown in Fig. 3: (1) Metallic seed layers $(10 \mathrm{~nm}$ Ti and $100 \mathrm{~nm} \mathrm{Cu}$ ) were sputtered on 4 inches oxidized silicon wafer $\left(\mathrm{SiO}_{2} / \mathrm{Si}\right)$ in the Denton Explorer 14 system; (2) The photoresist, AZ4562, was spin-coated on Si wafer, and then an UV exposure step was taken place by EVG 620 system through a photo-mask, and then this wafer was developed in AZ400K developer; (3) Copper was electroplated in a homemade $\mathrm{CuSO}_{4}$ solution bath under controlled current using AutoLab system (Metrohm); (4) The mold was removed with acetone; (5) The seed layer was etched by dry etching system (IBE - IonSys 500 (ROTH\&RAU)); (6) The planar coils were diced in suitable size and then they were ready for placing under PDMS channel (Fig. 4).

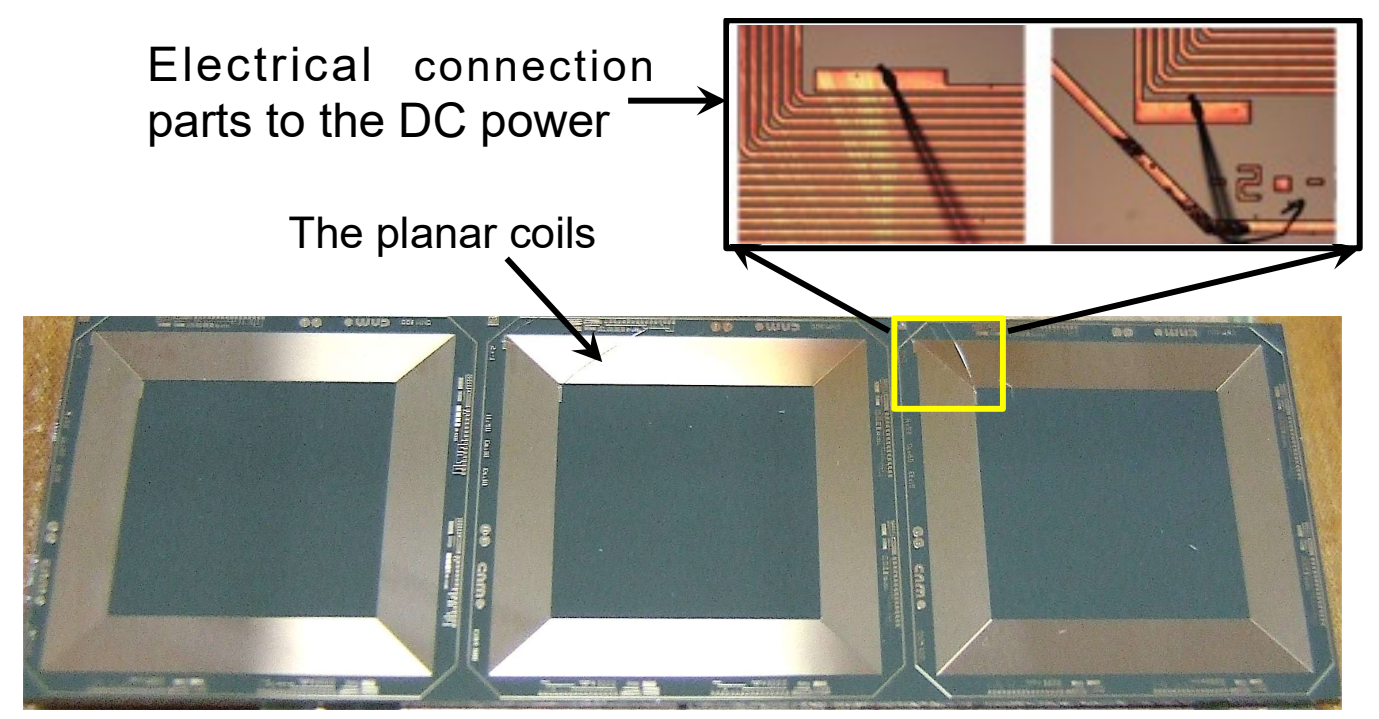

Fig. 4. Planar coils on silicon substrate at the final step.

\section{II.3. PDMS channel fabrication}

The microfluidic channel in PDMS materials was fabricated by replica-molding, and the process of fabrication consisted of two following steps: (1) Fabrication of the master mold: a $50 \mu \mathrm{m}$ thick layer of photoresist SU-8 3050 (Microchem) was spin-coated onto a silicon wafer ( 2 inches). An UV exposure with a transparency mask was carried out by the lithography technique; (2) A developing process was achieved by SU-8 developer solution and then rinsed by iso-propanol. A uniform mixture of the PDMS was prepared by mixing the pre-polymer with a curing agent (Sylgard 184, Dow Corning) at a ratio of 10:1, and then this fresh PDMS was poured in the SU-8 mold. The PDMS pre-polymer was cured at $90^{\circ} \mathrm{C}$ for 1 hour. The inlets and outlets of the channel were made by PDMS tool kits. Finally, embossed micro-channels were released from the master mold. Dimensions of the micro-channels are $50 \mu \mathrm{m}$ deep and $500 \mu \mathrm{m}$ wide and $3.5 \mathrm{~cm}$ long, Fig. 5. 

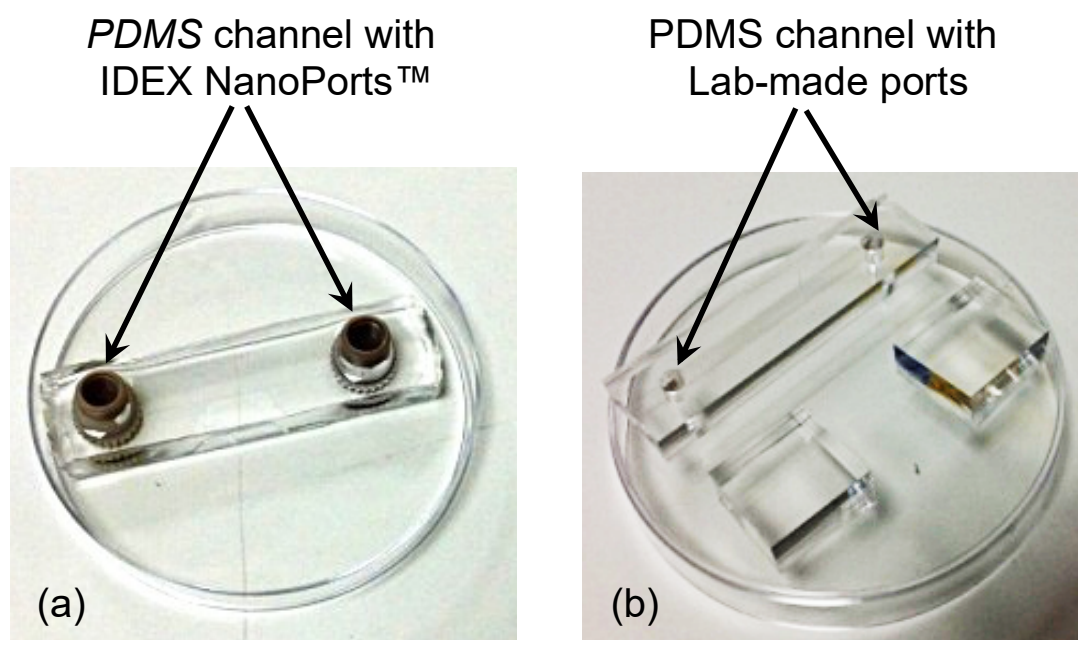

Fig. 5. (a). PDMS channel with the inlet and outlet were used the IDEX NanoPorts ${ }^{\mathrm{TM}}$; (b). PDMS channel with the inlet and outlet were drilled by PDMS tool kits.

\section{II.4. Assembling microfluidic chip}

The planar coil was fixed on PCB supporter, and the electric connection between coils and PCB was made by the aluminum wire micro-bonding. To protect the coil, a PDMS layer was fabricated on the top of the coil by spin-coating with $10 \mu \mathrm{m}$ thick. This layer is very important because it is not only an insulation layer but also a biocompatible layer and is bondable in packaging process. The results of magnetic field calculation (in the part 3.1) showed that a suitable thickness of PDMS layer had been met two requirements in protecting the coil from electric and obtaining an appropriate value of the magnetic force. The bonding of the channel cap to the coil was carried out by oxygen plasma treatment and cured in oven at $75^{\circ} \mathrm{C}$ in 1 hour. The obtained microfluidic chip was shown in Fig. 6.
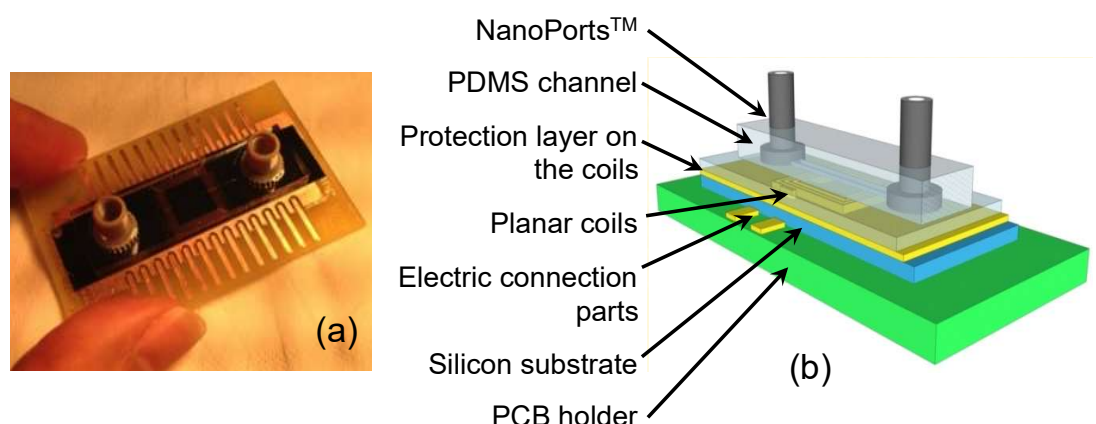

Fig. 6. (a). A picture of the microfluidic chip; (b). The structure layer of the microfluidic chip. 


\section{RESULT AND DISCUSSION}

\section{III.1. Magnetic field calculation}

The ANSYS ${ }^{\circledR}$ software was used to calculate the magnetic field generated by the planar coil. Due to the symmetry reason, only one half of a longitudinal cut was represented (Fig. 7). The 2D simulations were carried out with the assumption that the length of each segment is much larger than the useful working distance (from surface of coil to the position needed to calculate the magnetic field). Furthermore, in this case, the results obtained are valid whether the coil shape is circular or square.

The input parameters of coil for magnetic field calculation (Fig. 7): $L=10 \mu \mathrm{m} ; I=10 \mu \mathrm{m}$; $N=45$ turns; $R_{e x}=5000 \mu \mathrm{m}$, and value of $R_{c}$ was calculated from $R_{e x}, L, I, N$. Current density applied to the coil was assumed to be of $1.0 \times 10^{9} \mathrm{~A} / \mathrm{m}^{2}$.

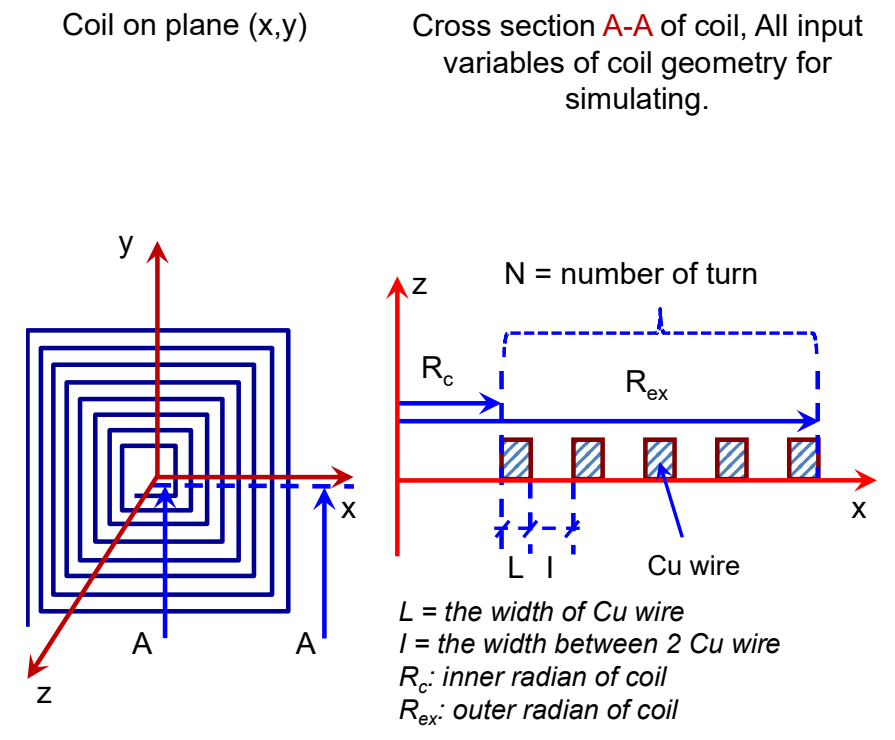

(a) (b)
The magnetic field at path 1 to 9

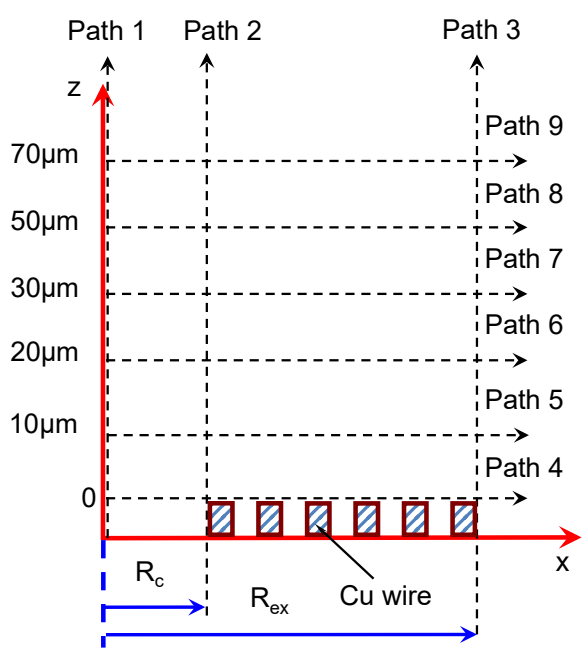

(c)

Fig. 7. The model of planar coil for the magnetic field calculation and simulation.

The calculation results of the magnetic field were shown in Fig. 8. The magnetic field fell off with the distance along $\mathrm{z}$-axis from the surface of coil increased corresponding to Path 4 - 9, (Fig. 7(c) and Fig. 8). At the distance farther than $50 \mu \mathrm{m}$ from the surface of the coil, the magnetic field was not significantly changed, Fig. 8(b). These results showed that in the process of fabricating the protection layer on surface of the coil it would be necessary to control the thickness of the PDMS layer to obtain the highest magnetic field. In the middle area of all wires (from the inner to the outer wire) the minimum value of the magnetic induction (B) was around $11.0 \mathrm{mT}$. The maximum value of $\mathrm{B}(\sim 11.8 \mathrm{mT})$ was found at the nearest wire to the coil center, these results were also shown at part 4 (Fig. 8(b)). This result was used to explain the result of trapping magnetic nanoparticles was significantly at the inner or outer wires (Fig. 11(b and c)). 


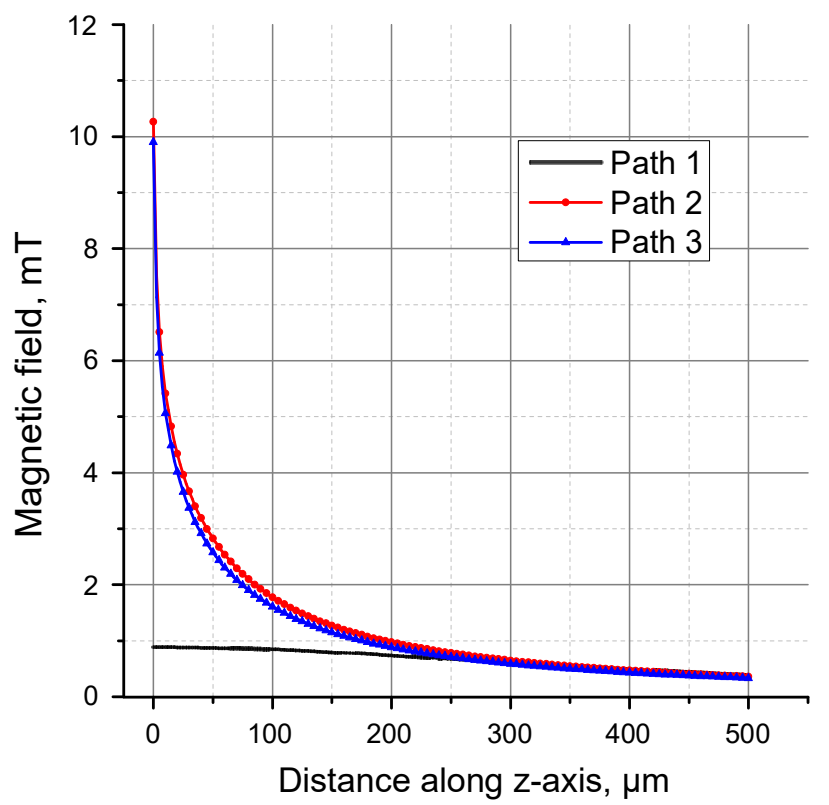

(a)

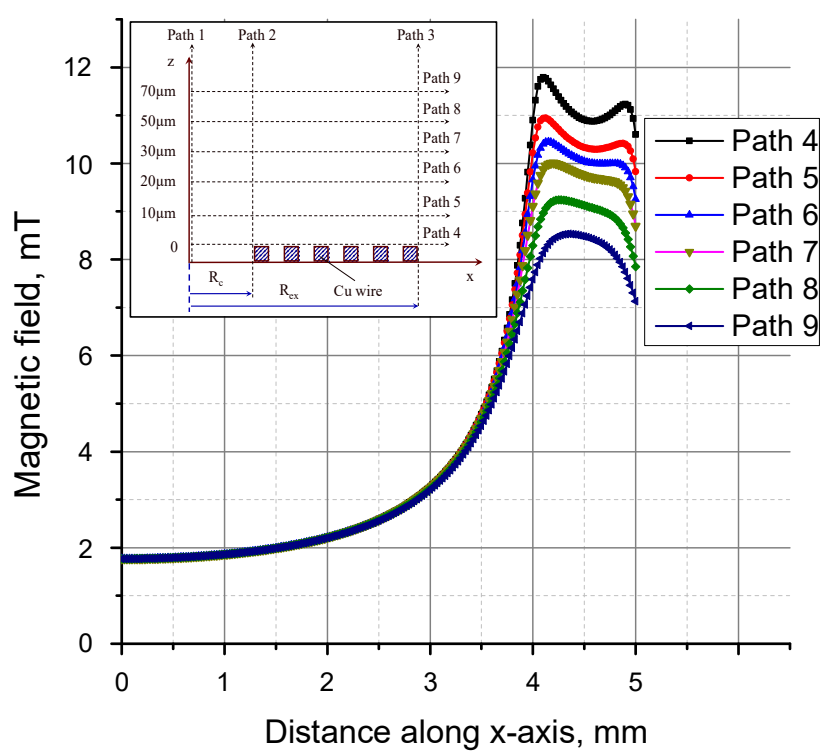

(b)

Fig. 8. The calculation of the magnetic induction (B) generating from the coil $(\mathrm{L}=10$ $\mu \mathrm{m} ; \mathrm{I}=10 \mu \mathrm{m} ; \mathrm{N}=45$ turns; $\mathrm{R}_{e x}=5000 \mu \mathrm{m}$ ) with the assumed current (I) of $100 \mathrm{~mA}$ was applied to the coil. (a) The changing of the magnetic induction (B) at parts 1,2, and 3 (shown in 5). (b) The changing of the magnetic field (B) at parts from 4 to 9. 


\section{III.2. Temperature measurement}

When a current was applied to the coil, the temperature on the surface of the coil increased due to the Joule effect. It means that the higher magnetic field value was generated by coil, the higher temperature on the surface of the coil was presented. This effect was very important to biological analyses and other application because it is required to apply a proper current to gain the most suitable the biocompatible temperature.

To measure the temperature on the top of coil surface and inside the channel during applying current to the coil, a small thermocouple (K type, $1 \mathrm{~mm}$ diameter of tip size) was put directly on the top of PDMS channel (Fig. 9).

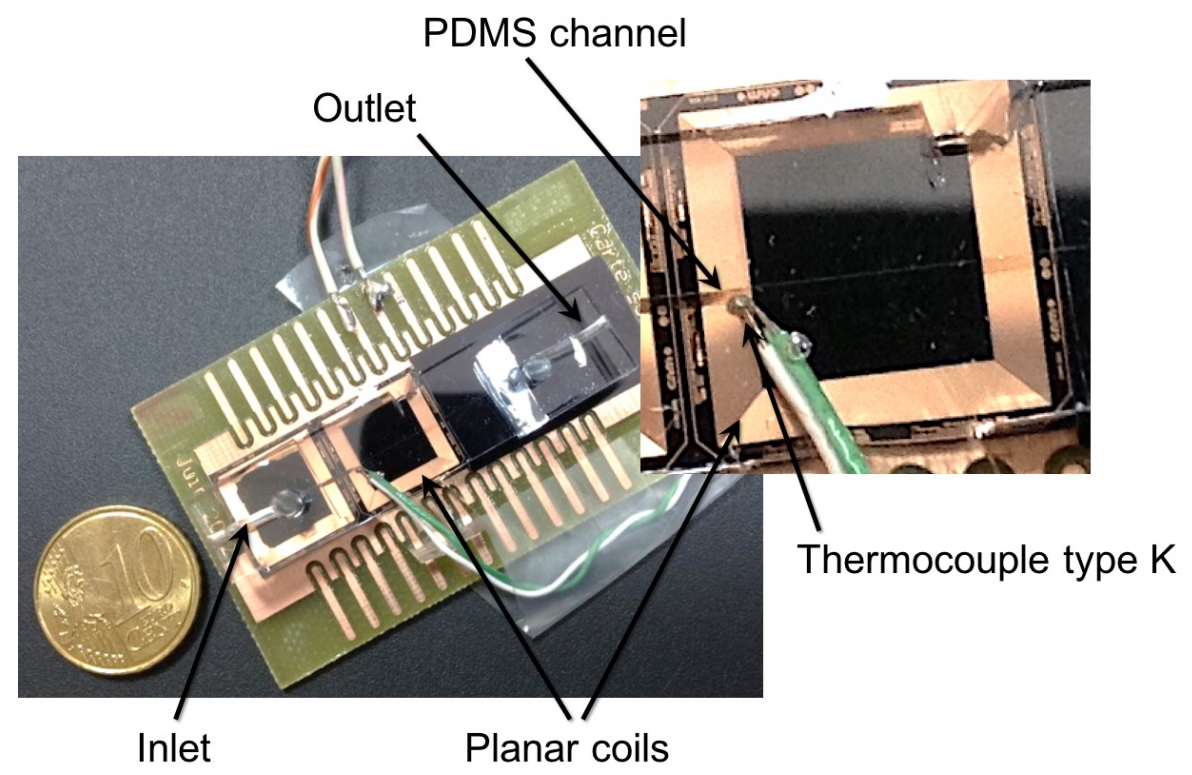

Fig. 9. The microfluidic chip with thermocouple on the top of channel.

The temperature was recorded by a digital multimeter during applying current to the coil. The current in the coil was controlled by DC power. Two experiments were carried out for this test: The temperature was measured in a microfluidics that was injected water into the channel (at a flow rate of $1.45 \mathrm{cc} / \mathrm{h}$ ) in the first experiment, and in other experiment, the water was not injected into the channel. The results of temperature measurement were shown in Fig. 10. The temperature profile inside the channel in these two tests had the same slope during applying current in two experiments.

Based on the study on the temperature measurement, the application of this integrated microfluidics can work at a suitable temperature depending on the applied current to coil. For example, in a biological analysis the temperature can be controlled under $37^{\circ} \mathrm{C}$, but in the trapping of magnetic nanoparticles only the temperature can be high. It implies that, it is necessary to optimize the current, temperature, and magnetic field value in experiments. 


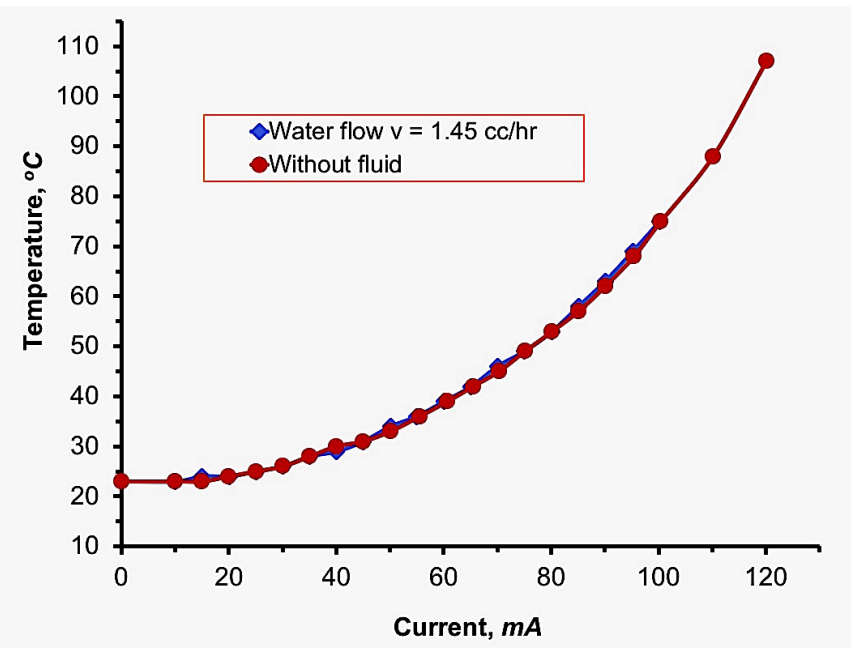

Fig. 10. The influence of applied current on temperature in a channel.

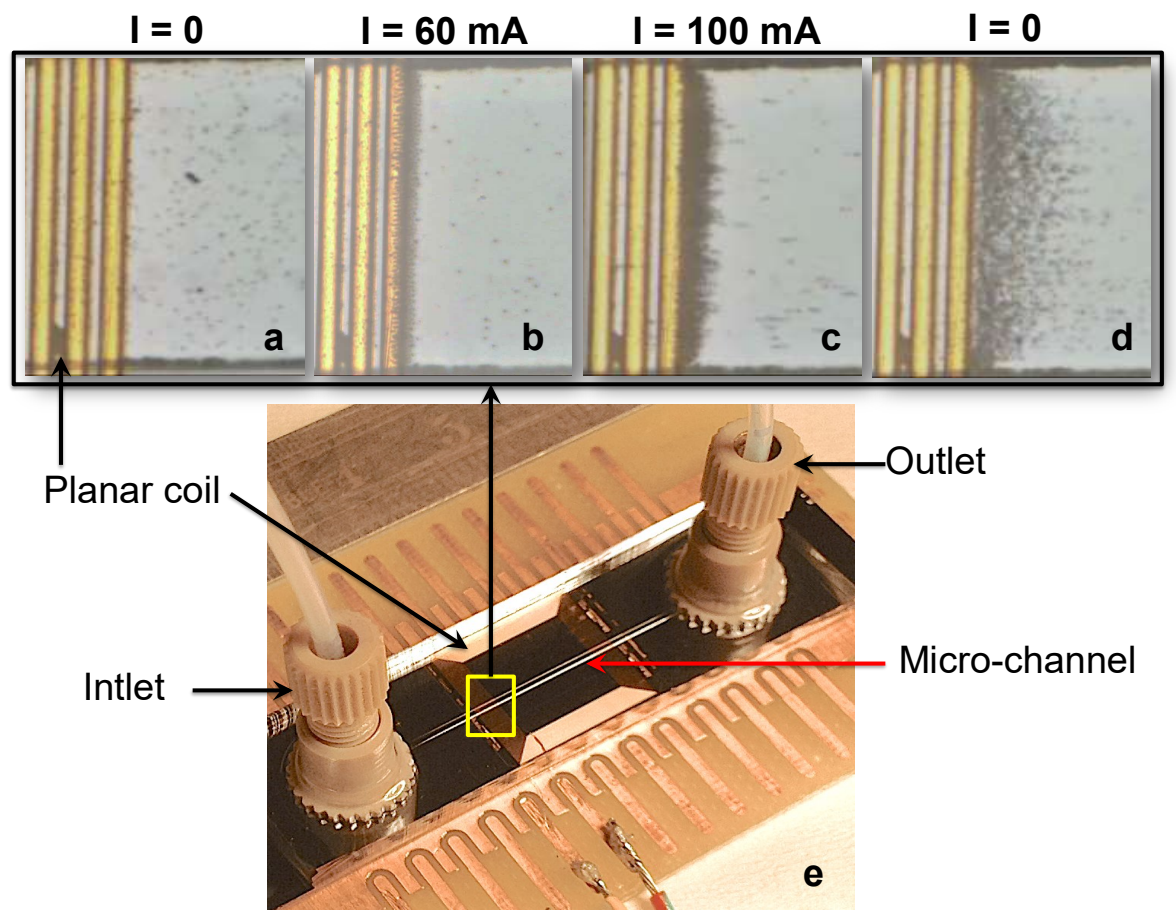

Fig. 11. Trapping magnetic particles in the microfluidics at different injected currents (I): (a) $\mathrm{I}=0 \mathrm{~mA}$; (b) $\mathrm{I}=100 \mathrm{~mA}$, (c) $\mathrm{I}=0 \mathrm{~mA}$. (d) Microfluidic chip. 


\section{III.3. Trapping magnetic nanoparticles}

In this study, commercial magnetic particles, Dynabeads ${ }^{\circledR}$ MyOne ${ }^{\mathrm{TM}}$ Carboxylic Acid with $1.05 \mu \mathrm{m}$ of diameter, were used for trapping test. These particles were dispersed in a phosphate saline buffer (PBS, $\mathrm{pH}=7.4$ ) at $10 \mathrm{mg} / \mathrm{L}$ of the concentration. The microfluidic chip integrated with a square coil ( $1 \mathrm{~cm}$ dimension, 45 turns, $10 \times 10 \mu \mathrm{m}$ size of copper wire) was fabricated for this test, Fig. 11(e). The liquid flow of dispersed particles was pumped into the channel (500 $\mu \mathrm{m}$ wide, $50 \mu \mathrm{m}$ high, and $3.5 \mathrm{~cm}$ long) at flow rate of $0.59 \mu \mathrm{L} / \mathrm{min}\left(3.45 \times 10^{-2} \mathrm{cc} / \mathrm{h}\right.$ ) by micro-syringe pump.

Figures 11(a-d) showed that, when a DC current was applied to the coil, the magnetic particles were trapped at the inner copper wire (Fig. 11(b)). When the current was off, the magnetic particles were released from the copper wire and flowed along the channel. The goal of this experiment was to find out how beads were trapped at the coil in a dynamic mode, continuous flow. Thus, a high current, $\mathrm{I}=100 \mathrm{~mA}$, was applied to the coil in the trapping process, the temperature inside channel was around $74^{\circ} \mathrm{C}$, Fig. 10 and Fig. $11(\mathrm{c})$. In case of the experiment working with biological agents, a suitable current, I ; $60 \mathrm{~mA}$, will be applied to the coil to avoid the temperature rising up $40^{\circ} \mathrm{C}$, Fig. $11(\mathrm{~b})$.

\section{CONCLUSIONS}

The microfluidic coil was fabricated and integrated in the microfluidic chip by microfabrication method. By calculating magnetic fields and measuring temperature inside the microchannel, it was proved that the planar coil could work well at low temperature $\left(<40^{\circ} \mathrm{C}\right)$ with the suitable value of magnetic field for trapping effectively the magnetic particles.

Base on this work, we will define the more characteristics of this microfluidic chip, and the more advanced tests in trapping functionalized magnetic nanoparticles with the aim of using in several kinds of biological analyses.

\section{ACKNOWLEDGMENTS}

This research was financially supported by the Vietnam National Foundation for Science and Technology Development (NAFOSTED); code: 104.99-2018.357.

The authors are grateful to Prof. Elisabeth Dufour-Gergam, and other members of the "MicroSystems and NanoBiofluidics" Department, The Center for Nanosciences and Nanotechnologies $(\mathrm{C} 2 \mathrm{~N})$. University Paris-Saclay for some experimental works.

\section{REFERENCES}

[1] H. Lee, Y. Liu, D. Ham, R. M. Westervelt, Lab on a chip 7 (2007) 331.

[2] N. Pamme, Lab on a chip 6 (2006) 24.

[3] M. A. M. Gijs, F. Lacharme, U. Lehmann, Chemical Reviews 110 (2010) 1518.

[4] G. Luka, A. Ahmadi, H. Najjaran, E. Alocilja, M. DeRosa, K. Wolthers, A. Malki, H. Aziz, A. Althani, M. Hoorfar, Sensors (Basel) 15 (2015) 30011.

[5] M. Herrmann, T. Veres, M. Tabrizian, Lab on a chip 6 (2006) 555.

[6] D. Holmes, J. K. She, P. L. Roach, H. Morgan, Lab on a chip 7 (2007) 1048.

[7] C. Quanliang, H. Xiaotao and L. Liang, J. Phys. D: Appl. Phys. 45 (2012) 465001.

[8] M. Shikida, M. Koyama, N. Nagao, R. Imai, H. Honda, M. Okochi, H. Tsuchiya, K. Sato, Sensors and Actuators B: Chemical 137 (2009) 774. 
[9] M. A. M. Gijs, Microfluidics and Nanofluidics 1 (2004) 22.

[10] H. Lee, A.M. Purdon, R.M. Westervelt, Applied Physics Letters 85 (2004) 1063.

[11] R. Fulcrand, A. Bancaud, C. Escriba, Q. He, S. Charlot, A. Boukabache, A.-M. Gué, Sensors and Actuators B: Chemical 160 (2011) 1520.

[12] K. Smistrup, O. Hansen, H. Bruus, M.F. Hansen, Journal of Magnetism and Magnetic Materials 293 (2005) 597.

[13] D. Issadore, T. Franke, K.A. Brown, R.M. Westervelt, Lab on a chip 10 (2010) 2937.

[14] S. Bronzeau, N. Pamme, Analytica Chimica Acta 609 (2008) 105.

[15] J. Peng, C. Fang, S. Ren, J. Pan, Y. Jia, Z. Shu, D. Gao, International Journal of Heat and Mass Transfer 130 (2019) 660.

[16] S. Babikian, L. Wu, G.P. Li, M. Bachman, 2012 IEEE 62nd Electronic Components and Technology Conference, 2012, pp. 1582-1587.

[17] Richard P. Feynman, Robert B. Leighton, M. Sands, The Feynman Lectures on Physics, New Millennium ed., Basic Books, New York, 2010. 